\title{
Injective objects in the category of finitely presented representations of an interval finite quiver
}

\author{
Pengjie Jiao
}

\begin{abstract}
We characterize the indecomposable injective objects in the category of finitely presented representations of an interval finite quiver.
\end{abstract}

\section{Introduction}

Infinite quivers appear naturally in the covering theory of algebras; see such as [BG82], [Gab81]. The injective representations of an infinite quiver $Q$ over an arbitrary ring $R$ is studied in [EEGR09]. We are interested in the category $\operatorname{fp}(Q)$ of finitely presented representations when $R$ is a field.

Recall that $\operatorname{fp}(Q)$ is studied in $[\mathrm{RVdB} 02]$ when $Q$ is locally finite of certain type. The result is used to classify the Noetherian Ext-finite hereditary abelian categories with Serre duality. More generally, when $Q$ is strongly locally finite, the Auslander-Reiten quiver of $\operatorname{fp}(Q)$ is studied in [BLP13]. The result is used to study the bounded derived category of a finite dimensional algebra with radical square zero in [BL17].

In the study of Auslander-Reiten theory of $\mathrm{fp}(Q)$, a natural question is how about the injective objects. We find that we can deal with it when $Q$ is interval finite (i.e., for any vertices $a$ and $b$, the set of paths from $a$ to $b$ is finite).

For each vertex $a$, we denote by $I_{a}$ the corresponding indecomposable injective representation. Let $p$ be a left infinite path, i.e., an infinite sequence of arrows $\ldots \alpha_{i} \ldots \alpha_{2} \alpha_{1}$ with $s\left(\alpha_{i+1}\right)=t\left(\alpha_{i}\right)$ for any $i \geq 1$. Denote by $[p]$ the equivalence class

Key words and phrases: indecomposable injective objects, finitely presented representations, interval finite quiver.

2010 Mathematics Subject Classification: 18G05, 16G20. 
(see page 390 for the definition) of left infinite paths containing $p$. Consider the indecomposable representation $Y_{[p]}$ introduced in [Jia19, Section 5]. We have that if $Y_{[p]}$ lies in $\operatorname{fp}(Q)$, then it is an indecomposable injective object; see Proposition 3.10.

Moreover, we can classify the indecomposable injective objects in $\operatorname{fp}(Q)$.

Main Theorem (see Theorem 3.11) Let $Q$ be an interval finite quiver. Assume $I$ is an indecomposable injective object in $\operatorname{fp}(Q)$. Then either $I \simeq I_{a}$ for certain vertex $a$, or $I \simeq Y_{[p]}$ for certain left infinite path $p$.

Compared with [Jia19, Theorem 6.8], the difficulty here is to characterize when $I_{a}$ and $Y_{[p]}$ are finitely presented. The result strengthens a description of finite dimensional indecomposable injective objects in $\mathrm{fp}(Q)$; see [BLP13, Proposition 1.16].

The paper is organized as follows. In Section 2, we recall some basic facts about quivers and representations. In Section 3, we study the injective objects in $\mathrm{fp}(Q)$ and give the classification theorem. Some examples are given in Section 4.

\section{Quivers and representations}

Let $k$ be a field, and $Q=\left(Q_{0}, Q_{1}\right)$ be a quiver, where $Q_{0}$ is the set of vertices and $Q_{1}$ is the set of arrows. For each arrow $\alpha: a \rightarrow b$, we denote by $s(\alpha)=a$ its source and by $t(\alpha)=b$ its target.

A path $p$ of length $l \geq 1$ is a sequence of arrows $\alpha_{l} \ldots \alpha_{2} \alpha_{1}$ such that $s\left(\alpha_{i+1}\right)=$ $t\left(\alpha_{i}\right)$ for any $1 \leq i \leq l-1$. We set $s(p)=s\left(\alpha_{1}\right)$ and $t(p)=t\left(\alpha_{l}\right)$. We associate each vertex $a$ with a trivial path (of length 0$) e_{a}$ with $s\left(e_{a}\right)=a=t\left(e_{a}\right)$. A nontrivial path $p$ is called an oriented cycle if $s(p)=t(p)$. For any $a, b \in Q_{0}$, we denote by $Q(a, b)$ the set of paths $p$ from $a$ to $b$, i.e., $s(p)=a$ and $t(p)=b$.

If $Q(a, b) \neq \varnothing$, then $a$ is called a predecessor of $b$, and $b$ is called a successor of $a$. For $a \in Q_{0}$, we denote by $a^{-}$the set of vertices $b$ with some arrow $b \rightarrow a$; by $a^{+}$ the set of vertices $b$ with some arrow $a \rightarrow b$.

A right infinite path $p$ is an infinite sequence of arrows $\alpha_{1} \alpha_{2} \ldots \alpha_{n} \ldots$ such that $s\left(\alpha_{i}\right)=t\left(\alpha_{i+1}\right)$ for any $i \geq 1$. We set $t(p)=t\left(\alpha_{1}\right)$. Dually, a left infinite path $p$ is an infinite sequence of arrows $\ldots \alpha_{n} \ldots \alpha_{2} \alpha_{1}$ such that $s\left(\alpha_{i+1}\right)=t\left(\alpha_{i}\right)$ for any $i \geq 1$. We set $s(p)=s\left(\alpha_{1}\right)$. Here, we use the terminologies in [Che15, Section 2.1]. We mention that these are opposite to the corresponding notions in [BLP13, Section 1].

A representation $M=(M(a), M(\alpha))$ of $Q$ over $k$ means a collection of $k$-linear spaces $M(a)$ for every $a \in Q_{0}$, and a collection of $k$-linear maps $M(\alpha): M(a) \rightarrow M(b)$ for every arrow $\alpha: a \rightarrow b$. For each nontrivial path $p=\alpha_{l} \ldots \alpha_{2} \alpha_{1}$, we denote $M(p)=$ $M\left(\alpha_{l}\right) \circ \ldots \circ M\left(\alpha_{2}\right) \circ M\left(\alpha_{1}\right)$. For each $a \in Q_{0}$, we set $M\left(e_{a}\right)=\mathbb{1}_{M(a)}$. A morphism $f: M \rightarrow N$ of representations is a collection of $k$-linear maps $f_{a}: M(a) \rightarrow N(a)$ for every $a \in Q_{0}$, such that $f_{b} \circ M(\alpha)=N(\alpha) \circ f_{a}$ for any arrow $\alpha: a \rightarrow b$. 
Let $\operatorname{Rep}(Q)$ be the category of representation of $Q$ over $k$. We denote by $\operatorname{Hom}(M, N)$ the set of morphisms from $M$ to $N$ in $\operatorname{Rep}(Q)$. It is well known that $\operatorname{Rep}(Q)$ is a hereditary abelian category; see [GR92, Section 8.2].

Recall that a subquiver $Q^{\prime}$ of $Q$ is called full if any arrow $\alpha$ with $s(\alpha), t(\alpha) \in$ $Q_{0}^{\prime}$ lies in $Q^{\prime}$. Let $M$ be a representation of $Q$. The support $\operatorname{supp} M$ of $M$ is the full subquiver of $Q$ formed by vertices $a$ with $M(a) \neq 0$. The socle $\operatorname{soc} M$ of $M$ is the subrepresentation such that $(\operatorname{soc} M)(a)=\bigcap_{\alpha \in Q_{1}, s(\alpha)=a} \operatorname{Ker} M(\alpha)$ for any vertex $a$. The radical $\operatorname{rad} M$ of $M$ is the subrepresentation such that $(\operatorname{rad} M)(a)=$ $\sum_{\alpha \in Q_{1}, t(\alpha)=a} \operatorname{Im} M(\alpha)$ for any vertex $a$.

We mention the following fact; see [BLP13, Lemma 1.1].

Lemma 2.1. If the support of a representation $M$ contains no left infinite paths, then soc $M$ is essential in $M$.

Proof. Let $N$ be a nonzero subrepresentation of $M$. Assume $x \in N(a)$ is nonzero for some vertex $a$. Since supp $M$ contains no left infinite paths, there exists some path $p$ in $\operatorname{supp} M$ with $s(p)=a$ such that $N(p)(x) \neq 0$ and $N(\alpha p)(x)=0$ for any arrow $\alpha$ in $Q$. Then $N(p)(x) \in(N \cap \operatorname{soc} M)(t(p))$. It follows that $\operatorname{soc} M$ is essential in $M$.

Let $a$ be a vertex in $Q$. We define a representation $P_{a}$ as follows. For every vertex $b$, we let

$$
P_{a}(b)=\bigoplus_{p \in Q(a, b)} k p .
$$

For every arrow $\alpha: b \rightarrow b^{\prime}$, we let

$$
P_{a}(\alpha): P_{a}(b) \longrightarrow P_{a}\left(b^{\prime}\right), p \longmapsto \alpha p .
$$

Similarly, we define a representation $I_{a}$ as follows. For every vertex $b$, we let

$$
I_{a}(b)=\operatorname{Hom}_{k}\left(\bigoplus_{p \in Q(b, a)} k p, k\right) .
$$

For every arrow $\alpha: b \rightarrow b^{\prime}$, we let

$$
I_{a}(\alpha): I_{a}(b) \longrightarrow I_{a}\left(b^{\prime}\right), \quad f \longmapsto(p \longmapsto f(p \alpha)) .
$$

The following result is well known; see [GR92, Section 3.7]. It implies that $P_{a}$ is a projective representation and $I_{a}$ is an injective representation in $\operatorname{Rep}(Q)$.

Lemma 2.2. Let $M \in \operatorname{Rep}(Q)$ and $a \in Q_{0}$. 
(1) The k-linear map

$$
\eta_{M}: \operatorname{Hom}\left(P_{a}, M\right) \longrightarrow M(a), f \longmapsto f_{a}\left(e_{a}\right),
$$

is an isomorphism natural in $M$.

(2) The k-linear map

$$
\zeta_{M}: \operatorname{Hom}\left(M, I_{a}\right) \longrightarrow \operatorname{Hom}_{k}(M(a), k), \quad f \longmapsto\left(x \longmapsto f_{a}(x)\left(e_{a}\right)\right)
$$

is an isomorphism natural in $M$.

Proof. (1) Consider the $k$-linear map

$$
\eta_{M}^{\prime}: M(a) \longrightarrow \operatorname{Hom}\left(P_{a}, M\right)
$$

given by $\left(\eta_{M}^{\prime}(x)\right)_{b}(p)=M(p)(x)$ for any $x \in M(a), b \in Q_{0}$ and $p \in Q(a, b)$. We observe that $\eta_{M}^{\prime} \circ \eta_{M}=\mathbb{1}_{\mathrm{Hom}\left(P_{a}, M\right)}$ and $\eta_{M} \circ \eta_{M}^{\prime}=\mathbb{1}_{M(a)}$. Then $\eta_{M}$ is an isomorphism.

(2) Consider the $k$-linear map

$$
\zeta_{M}^{\prime}: \operatorname{Hom}_{k}(M(a), k) \longrightarrow \operatorname{Hom}\left(M, I_{a}\right)
$$

given by $\left(\zeta_{M}^{\prime}(f)\right)_{b}(x)(p)=f(M(p)(x))$ for any $f \in \operatorname{Hom}_{k}(M(a), k), b \in Q_{0}, x \in M(b)$ and $p \in Q(b, a)$. We observe that $\zeta_{M}^{\prime} \circ \zeta_{M}=\mathbb{1}_{\operatorname{Hom}\left(M, I_{a}\right)}$ and $\zeta_{M} \circ \zeta_{M}^{\prime}=\mathbb{1}_{\operatorname{Hom}_{k}(M(a), k)}$. It follows that $\zeta_{M}$ is an isomorphism.

An epimorphism $P \rightarrow M$ with projective $P$ is called a projective cover of $M$ if it is an essential epimorphism. A monomorphism $M \rightarrow I$ with injective $I$ is called an injective envelope of $M$ if it is an essential monomorphism. We mention that two injective envelopes of $M$ are isomorphic.

Given a collection $\mathcal{A}$ of representations, we denote by add $\mathcal{A}$ the full subcategory of $\operatorname{Rep}(Q)$ formed by direct summands of finite direct sums of representations in $\mathcal{A}$. We set $\operatorname{proj}(Q)=\operatorname{add}\left\{P_{a} \mid a \in Q_{0}\right\}$ and $\operatorname{inj}(Q)=\operatorname{add}\left\{I_{a} \mid a \in Q_{0}\right\}$.

A representation $M$ is called finitely generated if there exists some epimorphism $f: \bigoplus_{i=1}^{n} P_{a_{i}} \rightarrow M$, and is called finitely presented if moreover Ker $f$ is also finitely generated. We denote by $\operatorname{fp}(Q)$ the subcategory of $\operatorname{Rep}(Q)$ formed by finitely presented representations.

We have the following well-known fact.

Proposition 2.3. The category $\mathrm{fp}(Q)$ is a hereditary abelian subcategory of $\operatorname{Rep}(Q)$ closed under extensions. 
Proof. Let $f: P \rightarrow P^{\prime}$ be a morphism in $\operatorname{proj}(Q)$. We observe that $\operatorname{Im} f$ is projective since $\operatorname{Rep}(Q)$ is hereditary. Then the induced exact sequence

$$
0 \longrightarrow \operatorname{Ker} f \longrightarrow P \longrightarrow \operatorname{Im} f \longrightarrow 0
$$

splits. Therefore Ker $f \in \operatorname{proj}(Q)$. It follows from [Aus66, Proposition 2.1] that $\operatorname{fp}(Q)$ is abelian. We observe by the horseshoe lemma that $\operatorname{fp}(Q)$ is closed under extensions in $\operatorname{Rep}(Q)$. In particular, it is hereditary.

We mention the following observation.

Lemma 2.4. Let $M$ be a finitely presented representation and $N$ be a finitely generated subrepresentation. Then $M / N$ is finitely presented.

Proof. Let $f: P \rightarrow M$ be an epimorphism with $P \in \operatorname{proj}(Q)$. Then $\operatorname{Ker} f$ is finitely generated. Denote by $g$ the composition of $f$ and the canonical surjection $M \rightarrow M / N$. Consider the following commutative diagram.

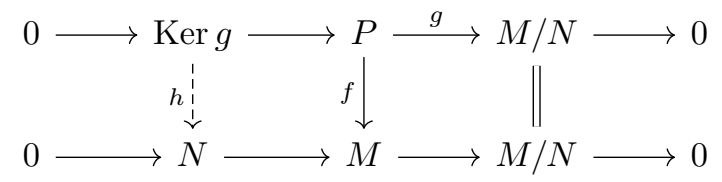

We observe that the left square is a pushout and also a pullback. Then $h$ is an epimorphism and Ker $h \simeq \operatorname{Ker} f$. In particular, Ker $h$ is finitely generated. Consider the exact sequence

$$
0 \longrightarrow \operatorname{Ker} h \longrightarrow \operatorname{Ker} g \stackrel{h}{\longrightarrow} N \longrightarrow 0 \text {. }
$$

It follows that Ker $g$ is finitely generated. Then $M / N$ is finitely presented.

The injective objects in $\operatorname{fp}(Q)$ satisfy the following property.

Lemma 2.5. Let $I$ be an injective object in $\mathrm{fp}(Q)$, and let $a \in Q_{0}$. Assume $p_{i}$ is a path from a to $b_{i}$ for $1 \leq i \leq n$ such that $p_{i}$ is not of the form up $p_{j}$ with $u \in Q\left(b_{j}, b_{i}\right)$ for any $j \neq i$. Then the $k$-linear map

$$
\left(\begin{array}{c}
I\left(p_{1}\right) \\
\vdots \\
I\left(p_{n}\right)
\end{array}\right): I(a) \longrightarrow \bigoplus_{i=1}^{n} I\left(b_{i}\right)
$$

is a surjection.

We mention that one can consider the special case that $p_{i}: a \rightarrow b_{i}$ for $1 \leq i \leq n$ are pairwise different arrows. 
Proof. We observe that the canonical morphism $\bigoplus_{i=1}^{n} P_{b_{i}} \rightarrow P_{a}$ induced by inclusions is a monomorphism. The injectivity of $I$ gives a surjection $\operatorname{Hom}\left(P_{a}, I\right) \rightarrow$ $\operatorname{Hom}\left(\bigoplus_{i=1}^{n} P_{b_{i}}, I\right)$. By identifying $\operatorname{Hom}\left(P_{c}, I\right)$ and $I(c)$ for any vertex $c$, we observe that the surjection is precisely the map needed.

The following fact is a direct consequence.

Corollary 2.6. The support of an injective object in $\mathrm{fp}(Q)$ is closed under predecessors.

Proof. Let $I$ be an injective object in $\operatorname{fp}(Q)$. Assume $a$ is a vertex in supp $I$ and $b \in a^{-}$. We can choose some arrow $\alpha: b \rightarrow a$. Lemma 2.5 implies that $I(\alpha)$ is a surjection. In particular, the vertex $b$ lies in $\operatorname{supp} I$. Then the result follows.

Recall that $Q$ is called interval finite if $Q(a, b)$ is finite for any $a, b \in Q_{0}$. A quiver is called top finite if there exist finitely many vertices of which every vertex is a successor, and is called socle finite if there exist finitely many vertices of which every vertex is a predecessor.

We have the following observation.

Lemma 2.7. A top finite interval finite quiver contains no right infinite paths; a socle finite interval finite quiver contains no left infinite paths.

Proof. Let $Q$ be a top finite interval finite quiver. Then there exist some vertices $b_{1}, b_{2}, \ldots, b_{n}$ such that any vertex is a successor of some $b_{i}$. Assume $Q$ contains a right infinite path $\alpha_{1} \alpha_{2} \ldots \alpha_{j} \ldots$. For each $j \geq 0$, we set $a_{j}=t\left(\alpha_{j+1}\right)$. Since $Q\left(b_{i}, a_{0}\right)$ is finite, there exists some nonnegative integer $Z_{i}$ such that $Q\left(b_{i}, a_{j}\right)=\varnothing$ for any $j \geq Z_{i}$. Let $Z=\max _{1 \leq i \leq n} Z_{i}$. Then $a_{Z}$ is not a successor of any $b_{i}$, which is a contradiction. It follows that $Q$ contains no right infinite paths.

Similarly, a socle finite interval finite quiver contains no left infinite paths.

\section{Finitely presented representations}

Let $k$ be a field and $Q$ be an interval finite quiver.

Recall that a representation $M$ is called pointwise finite dimensional if $M(a)$ is finite dimensional for any vertex $a$, and is called finite dimensional if moreover supp $M$ contains only finitely many vertices.

We mention the following fact.

Lemma 3.1. The abelian category $\mathrm{fp}(Q)$ is Hom-finite Krull-Schmidt, and every object is pointwise finite dimensional. 
Proof. Assume $\bigoplus_{i=1}^{m} P_{a_{i}} \rightarrow M$ is an epimorphism. We observe that each $P_{a_{i}}$ is pointwise finite dimensional, since $Q$ is interval finite. Then so is $M$.

Moreover, assume $\bigoplus_{j=1}^{n} P_{b_{j}} \rightarrow N$ is an epimorphism. Consider the maps

$$
\operatorname{Hom}(M, N) \hookrightarrow \operatorname{Hom}\left(\bigoplus_{i=1}^{m} P_{a_{i}}, N\right) \leftarrow \operatorname{Hom}\left(\bigoplus_{i=1}^{m} P_{a_{i}}, \bigoplus_{j=1}^{n} P_{b_{j}}\right) .
$$

We observe that $\operatorname{Hom}\left(\bigoplus_{i=1}^{m} P_{a_{i}}, \bigoplus_{j=1}^{n} P_{b_{j}}\right)$ is finite dimensional since $Q$ is interval finite. Then so is $\operatorname{Hom}(M, N)$. Therefore the abelian category $\operatorname{fp}(Q)$ is Hom-finite, and hence is Krull-Schmidt.

We need the following properties of finitely presented representations.

Lemma 3.2. Let $M$ be a finitely presented representation.

(1) $\operatorname{supp} M$ is top finite.

(2) $\bigcup_{a \in \operatorname{supp} M} a^{+} \backslash \operatorname{supp} M$ is finite.

Proof. (1) Assume $f: \bigoplus_{i=1}^{m} P_{a_{i}} \rightarrow M$ is an epimorphism. Then every vertex in $\operatorname{supp} M$ is a successor of some $a_{i}$. In other words, supp $M$ is top finite.

(2) Denote $\Delta=\bigcup_{a \in \operatorname{supp} M} a^{+} \backslash \operatorname{supp} M$. We observe that $\operatorname{Ker} f / \operatorname{rad} \operatorname{Ker} f$ is semisimple and $(\operatorname{Ker} f / \operatorname{rad} \operatorname{Ker} f)(b) \neq 0$ for any $b \in \Delta$. If $\Delta$ is not finite, then $\operatorname{Ker} f / \operatorname{rad} \operatorname{Ker} f$ is not finitely generated, which is a contradiction. It follows that $\Delta$ is finite.

Corollary 3.3. A finite dimensional representation $M$ is finitely presented if and only if $a^{+}$is finite for any vertex a in $\operatorname{supp} M$.

Proof. For the necessary, we assume $M$ is finitely presented and $a$ is a vertex in supp $M$. Lemma 3.2 implies that $a^{+} \backslash \operatorname{supp} M$ is finite. Since supp $M$ contains only finitely many vertices, then $a^{+} \cap \operatorname{supp} M$ is finite. It follows that $a^{+}$is finite.

For the sufficiency, we assume $a^{+}$is finite for any vertex $a$ in supp $M$. Since $M$ is finite dimensional, there exists some epimorphism $f: P \rightarrow M$ with $P \in \operatorname{proj}(Q)$.

Consider the subrepresentation $N$ of $P$ generated by $P(b)$, where $b$ runs over $\bigcup_{a \in \operatorname{supp} M} a^{+} \backslash \operatorname{supp} M$. Then $N$ is contained in Ker $f$. We observe by Lemma 3.1 each $P(b)$ is finite dimensional. Then $N$ is finitely generated.

Consider the factor module Ker $f / N$. Its support is contained in $\operatorname{supp} M$. Then it is finite dimensional and hence is finitely generated. Consider the exact sequence

$$
0 \longrightarrow N \longrightarrow \operatorname{Ker} f \longrightarrow \operatorname{Ker} f / N \longrightarrow 0
$$

It follows that Ker $f$ is finitely generated, and then $M$ is finitely presented. 
Corollary 3.4. Let a be a vertex. Then $I_{a}$ is finitely presented if and only if a admits only finitely many predecessors $b$ and each $b^{+}$is finite.

Proof. We observe that $I_{a}$ is finitely generated if and only if $\operatorname{supp} I$ contains only finitely many vertices, since $Q$ is interval finite. The vertices in $\operatorname{supp} I$ are precisely predecessors of $a$. Then the result follows from Corollary 3.3.

The support of an injective object in $\operatorname{fp}(Q)$ satisfies the following conditions.

Lemma 3.5. Let $I$ be an injective object in $\operatorname{fp}(Q)$.

(1) $a^{-} \cup a^{+}$is finite for any vertex a in $\operatorname{supp} I$.

(2) If supp I contains no left infinite paths, then it contains only finitely many vertices.

Proof. Lemma 3.2 implies that $\operatorname{supp} I$ is top finite. Then there exist some vertices $b_{1}, b_{2}, \ldots, b_{n}$ such that any vertex in supp $I$ is a successor of some $b_{i}$.

(1) We observe that supp $I$ is closed under predecessors; see Corollary 2.6. Then any vertex in $a^{-}$is a successor of some $b_{i}$. If $a^{-}$is infinite, then at least one $Q\left(b_{i}, a\right)$ is infinite, which is a contradiction. It follows that $a^{-}$is finite.

We observe that $a^{+} \cap \operatorname{supp} I$ is finite. Indeed, otherwise Lemma 2.5 implies that $I(a)$ is not finite dimensional, which is a contradiction. Since $a^{+} \backslash \operatorname{supp} I$ is finite by Lemma 3.2, then $a^{+}$is finite. It follows that $a^{-} \cup a^{+}$is finite.

(2) Assume the vertices in $\operatorname{supp} I$ is infinite. Then there exists some $b_{i}$ whose successors contained in $\operatorname{supp} I$ is infinite. Denote it by $a_{0}$. Since $a_{0}^{+} \cap \operatorname{supp} I$ is finite, then there exists some $a_{1} \in a_{0}^{+} \cap \operatorname{supp} I$ whose successors contained in supp $I$ is infinite. Choose some arrow $\alpha_{1}: a_{0} \rightarrow a_{1}$.

By induction, we obtain vertices $a_{i}$ and arrows $\alpha_{i+1}: a_{i} \rightarrow a_{i+1}$ for $i \geq 0$ in $\operatorname{supp} M$. This is a contradiction, since $\ldots \alpha_{i} \ldots \alpha_{2} \alpha_{1}$ is a left infinite path in supp $M$. It follows that supp $M$ contains only finitely many vertices.

For an injective object in $\operatorname{fp}(Q)$ whose support contains no left infinite paths, we have the following characterization.

Proposition 3.6. Let $I$ be an injective object in $\operatorname{fp}(Q)$ such that $\operatorname{supp} I$ contains no left infinite paths. Then

$$
I \simeq \bigoplus_{a \in Q_{0}} I_{a}^{\oplus \operatorname{dim}(\operatorname{soc} I)(a) .}
$$

Proof. It follows from Lemma 3.5 that supp $I$ contains only finitely many vertices. Let $J=\bigoplus_{a \in Q_{0}} I_{a}^{\oplus \operatorname{dim}(\operatorname{soc} I)(a)}$. This is a finite direct sum, since the vertices in supp $I$ are finite and $I$ is pointwise finite dimensional. 
For any vertex $a$ in $\operatorname{supp} I$, its predecessors are also contained in supp $I$; see Corollary 2.6. It follows that supp $J$ is a subquiver of $\operatorname{supp} I$. Then $\operatorname{soc} I$ and $J$ are finite dimensional. Corollary 3.3 implies that they are finitely presented. We observe by Lemma 2.1 that the inclusion $\operatorname{soc} I \subseteq I$ and the injection $\operatorname{soc} I \rightarrow J$ are injective envelopes in $\operatorname{fp}(Q)$. Then the result follows.

For an injective object in $\operatorname{fp}(Q)$ whose support contains some left infinite paths, we mention the following facts. They will be used technically in the proof of Theorem 3.11 .

Lemma 3.7. Let $I$ be an injective object in $\mathrm{fp}(Q)$, whose support contains some left infinite paths. Denote by $\Delta$ the set of left infinite paths $p$ contained in $\operatorname{supp} I$ with $s(p)^{-} \cap \operatorname{supp} I=\varnothing$. Then $\Delta$ is finite, and every left infinite path $p$ in supp $I$ admits some path $u$ with $p u \in \Delta$.

Proof. Lemma 3.2 implies that supp $I$ is top finite. Assume vertices $b_{1}, b_{2}, \ldots, b_{n}$ satisfy that any vertex in supp $I$ is a successor of some $b_{i}$. We can assume each $b_{i}^{-} \cap \operatorname{supp} I=\varnothing$.

Let $p$ be a left infinite path in $\operatorname{supp} I$. We observe that $s(p)$ is a successor of some $b_{i}$. Choose some $u \in Q\left(b_{i}, s(p)\right)$. Then $p u \in \Delta$. In particular, if $p \in \Delta$ then $b_{i}=s(p)$ since $s(p)^{-} \cap \operatorname{supp} I=\varnothing$.

Assume $\Delta$ is infinite. Then for $Z=\max _{1 \leq i \leq n} \operatorname{dim} I\left(b_{i}\right)$, One can find $n Z+1$ paths $u_{j}$ from some $b_{i}$ such that each $u_{j}$ is not of the form $v u_{j^{\prime}}$ for any $j^{\prime} \neq j$. We observe that at least one $1 \leq i \leq n$ such that the number of $u_{j}$ from $b_{i}$ is greater than $Z$. Then Lemma 2.5 implies that $\operatorname{dim} I\left(b_{i}\right)>Z$, which is a contradiction. Then the result follows.

Lemma 3.8. Let $I$ be an injective object in $\mathrm{fp}(Q)$, whose support contains some left infinite path $\ldots \alpha_{i} \ldots \alpha_{2} \alpha_{1}$. Set $a_{i}=s\left(\alpha_{i+1}\right)$ for any $i \geq 0$. Then there exists some nonnegative integer $Z$ such that $a_{i}^{+}=\left\{a_{i+1}\right\}, a_{i+1}^{-}=\left\{a_{i}\right\}$ and $I\left(\alpha_{i+1}\right)$ is a bijection for any $i \geq Z$.

Proof. We observe by Lemma 3.2 that supp $I$ is top finite and there exists some nonnegative integer $Z_{1}$ such that $a_{i}^{+}$is contained in supp $I$ for any $i \geq Z_{1}$.

It follows from Lemma 2.5 that $\operatorname{dim} I\left(a_{i}\right) \geq \operatorname{dim} I\left(a_{i+1}\right)$ for any $i \geq 0$. Then there exists some nonnegative integer $Z_{2} \geq Z_{1}$ such that $\operatorname{dim} I\left(a_{i}\right)=\operatorname{dim} I\left(a_{Z_{2}}\right)$ for any $i \geq Z_{2}$. Since $I\left(\alpha_{i+1}\right)$ is a surjection by Lemma 2.5 , then it is a bijection.

We claim that $a_{i}^{+}=\left\{a_{i+1}\right\}$ and $Q\left(a_{i}, a_{i+1}\right)=\left\{\alpha_{i+1}\right\}$ for any $i \geq Z_{2}$. Indeed, otherwise there exist some arrow $\beta: a_{i} \rightarrow b$ in $\operatorname{supp} I$ with $i \geq Z_{2}$ and $\beta \neq \alpha_{i+1}$. Then Lemma 2.5 implies that $\operatorname{dim} I\left(a_{i}\right) \geq \operatorname{dim} I\left(a_{i+1}\right)+\operatorname{dim} I(b)>\operatorname{dim} I\left(a_{i+1}\right)$, which is a contradiction. 
Assume vertices $b_{1}, b_{2}, \ldots, b_{n}$ satisfy that every vertex in $\operatorname{supp} I$ is a successor of some $b_{j}$. We observe that $\left|Q\left(b_{j}, a_{i+1}\right)\right| \geq\left|Q\left(b_{j}, a_{i}\right)\right|$ for any $1 \leq j \leq n$ and $i \geq 0$. If moreover $\left|a_{i+1}^{-}\right|>1$ for some $i \geq 0$, then there exists some $j$ such that $\left|Q\left(b_{j}, a_{i+1}\right)\right|>$ $\left|Q\left(b_{j}, a_{i}\right)\right|$.

We claim the existence of nonnegative integer $Z_{3}$ such that $a_{i+1}^{-}=\left\{a_{i}\right\}$ for any $i \geq Z_{3}$. Indeed, otherwise there exists some $1 \leq j \leq n$ such that $\left\{\left|Q\left(b_{j}, a_{i}\right)\right| \mid i \geq 0\right\}$ is unbounded. Then Lemma 2.5 implies that $I\left(b_{j}\right)$ is not finite dimensional, which is a contradiction.

Let $Z=\max \left\{Z_{2}, Z_{3}\right\}$. Then the result follows.

Following [Che15, Subsection 2.1], we define an equivalence relation on left infinite paths. Two left infinite paths $\ldots \alpha_{i} \ldots \alpha_{2} \alpha_{1}$ and $\ldots \beta_{i} \ldots \beta_{2} \beta_{1}$ are equivalent if there exist some positive integers $m$ and $n$ such that

$$
\ldots \alpha_{i} \ldots \alpha_{m+1} \alpha_{m}=\ldots \beta_{i} \ldots \beta_{n+1} \beta_{n} .
$$

Let $p$ be a left infinite path. We denote by $[p]$ the equivalence class containing $p$. We mention that $[p]$ is a set. For any vertex $a$, we denote by $[p]_{a}$ the subset of $[p]$ formed by left infinite paths $u$ with $s(u)=a$.

Considering [Jia19, Section 5] and [Che15, Subsection 3.1], we introduce a representation $Y_{[p]}$ as follows. For every vertex $a$, we let

$$
Y_{[p]}(a)=\operatorname{Hom}_{k}\left(\bigoplus_{u \in[p]_{a}} k u, k\right) .
$$

For every arrow $\alpha: a \rightarrow b$, we let

$$
Y_{[p]}(\alpha): Y_{[p]}(a) \longrightarrow Y_{[p]}(b), \quad f \longmapsto(u \longmapsto f(u \alpha))
$$

We mention that these $Y_{[p]}$ are indecomposable and pairwise non-isomorphic; see the dual of [Jia19, Proposition 5.4].

Recall that a quiver is called uniformly interval finite, if there exists some positive integer $Z$ such that for any vertices $a$ and $b$, the number of paths $p$ from $a$ to $b$ is less than or equal to $Z$; see [Jia19, Definition 2.3].

We characterize when $Y_{[p]}$ is finitely presented.

Lemma 3.9. Let $p$ be a left infinite path. Then $Y_{[p]}$ is finitely presented if and only if supp $Y_{[p]}$ is top finite uniformly interval finite and $\bigcup_{a \in \operatorname{supp} Y_{[p]}} a^{+} \backslash \operatorname{supp} Y_{[p]}$ is finite. 
Proof. For the necessary, we assume $Y_{[p]}$ is finitely presented. It follows from Lemma 3.2 that $\operatorname{supp} Y_{[p]}$ is top finite and $\bigcup_{a \in \operatorname{supp} Y_{[p]}} a^{+} \backslash \operatorname{supp} Y_{[p]}$ is finite.

Assume vertices $b_{1}, b_{2}, \ldots, b_{n}$ satisfy that any vertex in $\operatorname{supp} Y_{[p]}$ is a successor of some $b_{i}$. Let $a$ and $a^{\prime}$ be a pair of vertices in $\operatorname{supp} Y_{[p]}$. Then there exists some $Q\left(b_{i}, a\right) \neq \varnothing$. Set $Z=\max _{1 \leq i \leq n} \operatorname{dim} Y_{[p]}\left(b_{i}\right)$. Since $Q$ contains no oriented cycles, we have that

$$
\left|Q\left(a, a^{\prime}\right)\right| \leq\left|Q\left(b_{i}, a^{\prime}\right)\right| \leq\left|[p]_{b_{i}}\right|=\operatorname{dim} Y_{[p]}\left(b_{i}\right) \leq Z \text {. }
$$

It follows that $\operatorname{supp} Y_{[p]}$ is uniformly interval finite.

For the sufficiency, we assume $p=\ldots \alpha_{i} \ldots \alpha_{2} \alpha_{1}$. Set $a_{i}=s\left(\alpha_{i+1}\right)$ for any $i \geq 0$. Assume vertices $b_{1}, b_{2}, \ldots, b_{n}$ satisfy that any vertex in $\operatorname{supp} Y_{[p]}$ is a successor of some $b_{i}$. Since $\operatorname{supp} Y_{[p]}$ is uniformly interval finite, then

$$
\left\{\left|Q\left(b_{j}, a_{i}\right)\right| \mid i \geq 0,1 \leq j \leq n\right\}
$$

is bounded. We observe that $\left|Q\left(b_{j}, a_{i}\right)\right| \leq\left|Q\left(b_{j}, a_{i+1}\right)\right|$. Then there exists some nonnegative integer $Z_{1}$ such that $\left|Q\left(b_{j}, a_{i}\right)\right|=\left|Q\left(b_{j}, a_{Z_{1}}\right)\right|$ for any $i \geq Z_{1}$ and $1 \leq j \leq n$. In particular, $a_{i+1}^{-}=\left\{a_{i}\right\}$ and $Q\left(a_{i}, a_{i+1}\right)=\left\{\alpha_{i+1}\right\}$ for any $i \geq Z_{1}$.

Since $\bigcup_{a \in \operatorname{supp} Y_{[p]}} a^{+} \backslash \operatorname{supp} Y_{[p]}$ is finite, there exists some nonnegative integer $Z_{2}$ such that $a_{i}^{+}$is contained in $\operatorname{supp} Y_{[p]}$ for any $i \geq Z_{2}$. Let $Z=\max \left\{Z_{1}, Z_{2}\right\}$. Then $a_{i+1}^{-}=\left\{a_{i}\right\}, a_{i}^{+}=\left\{a_{i+1}\right\}$ and $Y_{[p]}\left(\alpha_{i+1}\right)$ is a bijection.

Consider the subrepresentation $N$ of $Y_{[p]}$ generated by $Y_{[p]}\left(a_{Z}\right)$. We observe that $N \simeq P_{a_{Z}}^{\oplus \operatorname{dim} Y_{[p]}\left(a_{Z}\right)}$ and hence is finitely presented. Moreover, $\operatorname{supp}\left(Y_{[p]} / N\right)$ contains only finitely many vertices $b$ and $b^{+}$is finite. Then $Y_{[p]} / N$ is finitely presented by Corollary 3.3. Consider the exact sequence

$$
0 \longrightarrow N \longrightarrow Y_{[p]} \longrightarrow Y_{[p]} / N \longrightarrow 0 .
$$

It follows that $Y_{[p]}$ is finitely presented.

We show the injectivity of finitely presented $Y_{[p]}$ in $\operatorname{fp}(Q)$; compare the dual of [Jia19, Proposition 6.2].

Proposition 3.10. Let $p$ be a left infinite path such that $Y_{[p]}$ is finitely presented. Then $Y_{[p]}$ is an indecomposable injective object in $\operatorname{fp}(Q)$.

Proof. Assume $p=\ldots \alpha_{i} \ldots \alpha_{2} \alpha_{1}$. For each $i \geq 0$, we set $a_{i}=s\left(\alpha_{i+1}\right)$. Consider the morphism $\psi_{i+1}: I_{a_{i+1}} \rightarrow I_{a_{i}}$ given by $\left(\psi_{i+1}\right)_{b}(f)(u)=f\left(\alpha_{i+1} u\right)$ for any $f \in I_{a_{i+1}}(b)$ and $u \in Q\left(b, a_{i}\right)$. We observe that $\left(I_{a_{i}}\right)_{i \geq 0}$ forms an inverse system, and $Y_{[p]}$ is the inverse limit in $\operatorname{Rep}(Q)$; see also [Jia19, Lemma 5.7].

Given any exact sequence

$$
0 \longrightarrow L \longrightarrow M \longrightarrow N \longrightarrow 0
$$


in $\operatorname{fp}(Q)$, it is also an exact sequence in $\operatorname{Rep}(Q)$. Applying $\operatorname{Hom}\left(-, I_{a_{i}}\right)$, we obtain an exact sequence of inverse systems of $k$-linear spaces

$$
0 \longrightarrow\left(\operatorname{Hom}\left(N, I_{a_{i}}\right)\right) \longrightarrow\left(\operatorname{Hom}\left(M, I_{a_{i}}\right)\right) \longrightarrow\left(\operatorname{Hom}\left(L, I_{a_{i}}\right)\right) \longrightarrow 0 .
$$

Lemma 2.2 implies that $\operatorname{Hom}\left(N, I_{a_{i}}\right) \simeq \operatorname{Hom}_{k}\left(N\left(a_{i}\right), k\right)$. We observe by Lemma 3.1 that it is finite dimensional. Then $\left(\operatorname{Hom}\left(N, I_{a_{i}}\right)\right)$ satisfies the Mittag-Leffler condition naturally. It follows from [Wei94, Proposition 3.5.7] the exact sequence

$$
0 \longrightarrow \lim _{\longleftarrow} \operatorname{Hom}\left(N, I_{a_{i}}\right) \longrightarrow \lim _{\longleftarrow} \operatorname{Hom}\left(M, I_{a_{i}}\right) \longrightarrow \lim _{\longleftarrow} \operatorname{Hom}\left(L, I_{a_{i}}\right) \longrightarrow 0 .
$$

For any $X \in \mathrm{fp}(Q)$, there exist natural isomorphisms

$$
\underset{\lim }{\longleftarrow} \operatorname{Hom}\left(X, I_{a_{i}}\right) \simeq \operatorname{Hom}\left(X, \lim _{\longleftarrow} I_{a_{i}}\right) \simeq \operatorname{Hom}\left(X, Y_{[p]}\right) .
$$

Then we obtain the exact sequence

$$
0 \longrightarrow \operatorname{Hom}\left(N, Y_{[p]}\right) \longrightarrow \operatorname{Hom}\left(M, Y_{[p]}\right) \longrightarrow \operatorname{Hom}\left(L, Y_{[p]}\right) \longrightarrow 0
$$

It follows that $Y_{[p]}$ is an indecomposable injective object in $\operatorname{fp}(Q)$.

Now, we can classify the indecomposable injective objects in $\mathrm{fp}(Q)$.

Theorem 3.11. Let $Q$ be an interval finite quiver. Assume $I$ is an indecomposable injective object in $\operatorname{fp}(Q)$. Then either $I \simeq I_{a}$ where a admits only finitely many predecessors $b$ and each $b^{+}$is finite, or $I \simeq Y_{[p]}$ where $[p]$ is an equivalence class of left infinite paths such that $\operatorname{supp} Y_{[p]}$ is top finite uniformly interval finite and $\bigcup_{a \in \operatorname{supp} Y_{[p]}} a^{+} \backslash \operatorname{supp} Y_{[p]}$ is finite.

Proof. If supp $I$ contains no left infinite paths, Proposition 3.6 implies that $I \simeq I_{a}$ for some vertex $a$. Corollary 3.4 implies that $a$ admits only finitely many predecessors $b$ and each $b^{+}$is finite.

Now, we assume supp $I$ contains some left infinite paths. Let $\Delta$ be the set of left infinite paths $p$ contained in $\operatorname{supp} I$ with $s(p)^{-} \cap \operatorname{supp} I=\varnothing$. It follows from Lemma 3.7 that $\Delta$ is finite.

For every $p \in \Delta$, we assume $p=\ldots \alpha_{p, j} \ldots \alpha_{p, 2} \alpha_{p, 1}$. Set $a_{p, j}=s\left(\alpha_{p, j+1}\right)$ for each $j \geq 0$. By Lemma 3.8, there exists some nonnegative integer $Z_{p}$ such that $a_{p, j}^{+}=$ $\left\{a_{p, j+1}\right\}, a_{p, j+1}^{-}=\left\{a_{p, j}\right\}$ and $I\left(\alpha_{p, j+1}\right)$ is a bijection for any $j \geq Z_{p}$.

Consider the subrepresentation $M$ of $I$ generated by $I\left(a_{p, Z_{p}+1}\right)$ for all $p \in \Delta$. It follows from Lemma 2.4 that $I / M$ is finitely presented. It is an injective object in $\operatorname{fp}(Q)$, since $\operatorname{fp}(Q)$ is hereditary.

We observe by Lemma 3.7 that $\operatorname{supp}(I / M)$ contains no left infinite paths. Indeed, assume $\ldots \alpha_{i} \ldots \alpha_{2} \alpha_{1}$ is a left infinite path in $\operatorname{supp}(I / M)$. It also lies in 
supp $I$. Then these $\alpha_{i}$ for $i$ large enough lie in supp $M$. Therefore, they do not lie in $\operatorname{supp}(I / M)$, which is a contradiction.

It follows from Proposition 3.6 that

$$
I / M \simeq \bigoplus_{b \in Q_{0}} I_{b}^{\oplus \operatorname{dim}(\operatorname{soc}(I / M))(b)} .
$$

For any $p \in \Delta$, we observe that $(\operatorname{soc}(I / M))\left(a_{p, Z_{p}}\right)=I\left(a_{p, Z_{p}}\right) \neq 0$ and $I\left(\alpha_{p, j+1}\right)$ is a bijection for any $j \geq Z_{p}$. The previous isomorphism can be extended as

$$
I \simeq\left(\bigoplus_{b \in Q_{0}} I_{b}^{\oplus \operatorname{dim}(\operatorname{soc} I)(b)}\right) \oplus\left(\bigoplus_{p \in \Delta} Y_{[p]}^{\oplus \operatorname{dim} I\left(a_{p}, Z_{p}\right)}\right) .
$$

Since $I$ is indecomposable, then $\Delta$ contains only one left infinite path $p$ and $\operatorname{dim} I\left(a_{p, Z_{p}}\right)=1$. Then soc $I=0$ and $I \simeq Y_{[p]}$. It follows from Lemma 3.9 that $\operatorname{supp} Y_{[p]}$ is top finite and uniformly interval finite, and $\bigcup_{a \in \operatorname{supp} Y_{[p]}} a^{+} \backslash \operatorname{supp} Y_{[p]}$ is finite.

Remark 3.12. Let $\mathcal{C}$ be a $k$-linear spectroid, i.e., a Hom-finite category whose objects are pairwise non-isomorphic with local endomorphism rings. Assume $k$ is algebraically closed, and the infinite radical of $\mathcal{C}$ vanishes, and the category of modules over $\mathcal{C}$ is hereditary. Then the quiver of $\mathcal{C}$ is interval finite. It can be viewed as a category naturally, and its $k$-linearization is precisely $\mathcal{C}$; see [GR92, Sections 8.1 and 8.2] for more details. Therefore, Theorem 3.11 can be applied to the category of finitely presented modules over $\mathcal{C}$.

\section{Examples}

Let $k$ be a field. We will give some examples.

Example 4.1. Assume $Q$ is the following quiver.

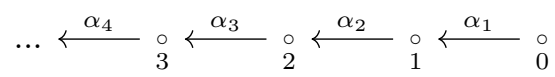

For each $n \geq 0$, we consider the representation $I_{n}$. We observe that the predecessors of $n$ are $i$ for $0 \leq i \leq n$, and $i^{+}$is finite. Corollary 3.4 implies that $I_{n}$ is finitely presented.

Let $p=\ldots \alpha_{i} \ldots \alpha_{2} \alpha_{1}$. Then $\operatorname{supp} Y_{[p]}=Q$. We observe that $\operatorname{supp} Y_{[p]}$ is top finite uniformly interval finite and $\bigcup_{a \in Q_{0}} a^{+} \backslash Q_{0}$ is the empty set. Lemma 3.9 implies that $Y_{[p]}$ is finitely presented.

We observe by Theorem 3.11 that

$$
\left\{I_{n} \mid n \geq 0\right\} \cup\left\{Y_{[p]}\right\}
$$

is a complete set of indecomposable injective objects in $\operatorname{fp}(Q)$. 
Example 4.2. Assume $Q$ is the following quiver.

$$
\ldots \stackrel{\alpha_{2}}{\leftarrow} \stackrel{\alpha_{1}}{\longleftarrow} \stackrel{\circ}{\longleftarrow} \stackrel{\alpha_{0}}{\longleftarrow} \underset{-1}{\longleftarrow} \stackrel{\alpha_{-1}}{\longleftarrow} \ldots
$$

For each integer $n$, we consider the representation $I_{n}$. We observe that all $i \leq n$ are predecessors of $n$. Corollary 3.4 implies that $I_{n}$ is not finitely presented.

Let $p=\ldots \alpha_{i} \ldots \alpha_{2} \alpha_{1}$. Then $\operatorname{supp} Y_{[p]}=Q$, which contains a right infinite path $\alpha_{-1} \alpha_{-2} \ldots \alpha_{-i} \ldots$ Then it is not top finite by Lemma 2.7. It follows from Lemma 3.9 that $Y_{[p]}$ is not finitely presented.

We observe by Theorem 3.11 that $\operatorname{fp}(Q)$ contains no nonzero injective objects.

Example 4.3. Assume $Q$ is the following quiver.

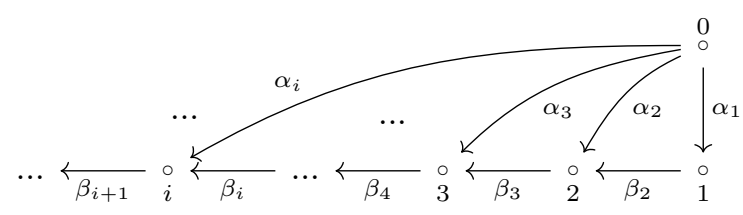

We mention that $Q$ is interval finite, but not locally finite (i.e., for any vertex $a$, the set of arrows $\alpha$ with $s(\alpha)=a$ or $t(\alpha)=a$ is finite).

For each $n \geq 0$, we consider the representation $I_{n}$. We observe that the set of predecessors of $n$ is $\{0 \leq i \leq n\}$, but $0^{+}$is not finite. Then Corollary 3.4 implies that $I_{n}$ is not finitely presented.

Let $p=\ldots \beta_{i} \ldots \beta_{3} \beta_{2}$. Then $\operatorname{supp} Y_{[p]}=Q$, which is not uniformly interval finite. Lemma 3.9 implies that $Y_{[p]}$ is not finitely presented.

We observe by Theorem 3.11 that $\operatorname{fp}(Q)$ contains no nonzero injective objects.

Example 4.4. Assume $Q$ is the following quiver.

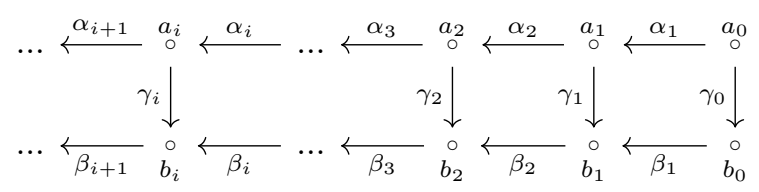

For each $n \geq 0$, we consider the representations $I_{a_{n}}$ and $I_{b_{n}}$. We observe that the set of predecessors of $a_{n}$ is $\left\{a_{i} \mid 0 \leq i \leq n\right\}$, and the one of $b_{n}$ is $\left\{a_{i} \mid 0 \leq i \leq n\right\} \cup$ $\left\{b_{i} \mid 0 \leq i \leq n\right\}$. Since each $a_{i}^{+}$and $b_{i}^{+}$are both finite, Corollary 3.4 implies that $I_{a_{n}}$ and $I_{b_{n}}$ are finitely presented.

Let $p=\ldots \alpha_{i} \ldots \alpha_{2} \alpha_{1}$. Then $\operatorname{supp} Y_{[p]}$ is the full subquiver of $Q$ formed by $a_{i}$ for all $i \geq 0$. We observe that $\bigcup_{a \in \operatorname{supp} Y_{[p]}} a^{+} \backslash \operatorname{supp} Y_{[p]}$ contains all $b_{i}$ and then is infinite. Lemma 3.9 implies that $Y_{[p]}$ is not finitely presented. 
Let $q=\ldots \beta_{i} \ldots \beta_{2} \beta_{1}$. Then $\operatorname{supp} Y_{[q]}=Q$. Since $Q$ is not uniformly interval finite, then $Y_{[q]}$ is not finitely presented by Lemma 3.9 .

We observe that $\{[p],[q]\}$ is the set of equivalence classes of left infinite paths. It follows from Theorem 3.11 that

$$
\left\{I_{a_{i}} \mid i \geq 0\right\} \cup\left\{I_{b_{i}} \mid i \geq 0\right\}
$$

is a complete set of indecomposable injective objects in $\operatorname{fp}(Q)$.

Example 4.5. Assume $Q$ is the following quiver.

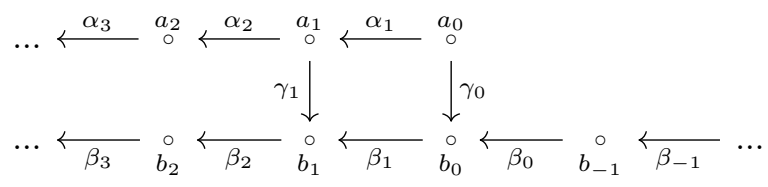

We observe that $a^{+}$is finite for any $a \in Q_{0}$. Consider the representations $I_{a_{i}}$ for $i \geq 0$ and $I_{b_{j}}$ for $j \in \mathbb{Z}$. The set of predecessors of $a_{i}$ is finite and the one of $b_{j}$ is not. It follows from Corollary 3.4 that $I_{a_{i}}$ is finitely presented, while $I_{b_{j}}$ is not.

Let $p=\ldots \alpha_{i} \ldots \alpha_{2} \alpha_{1}$. Then $\operatorname{supp} Y_{[p]}$ is the full subquiver of $Q$ formed by $a_{i}$ for all $i \geq 0$. We observe that $\operatorname{supp} Y_{[p]}$ is top finite uniformly interval finite, and $\bigcup_{a \in \operatorname{supp} Y_{[p]}} a^{+} \backslash \operatorname{supp} Y_{[p]}=\left\{b_{0}, b_{1}\right\}$. It follows from Lemma 3.9 that $Y_{[p]}$ is finitely presented.

Let $q=\ldots \beta_{i} \ldots \beta_{2} \beta_{1}$. Then supp $Y_{[q]}$ is the full subquiver of $Q$ formed by $a_{0}$, $a_{1}$ and $b_{j}$ for all $j \in \mathbb{Z}$. We observe that $\operatorname{supp} Y_{[q]}$ contains a right infinite path $\beta_{-1} \beta_{-2} \ldots \beta_{-i} \ldots$. Then it is not top finite by Lemma 2.7. It follows from Lemma 3.9 that $Y_{[q]}$ is not finitely presented.

We observe that $\{[p],[q]\}$ is the set of equivalence classes of left infinite paths. It follows from Theorem 3.11 that

$$
\left\{I_{a_{i}} \mid i \geq 0\right\} \cup\left\{Y_{[p]}\right\}
$$

is a complete set of indecomposable injective objects in $\operatorname{fp}(Q)$.

Acknowledgements. The author is very grateful to the referee for many helpful suggestions and comments, especially the spectroid point of view in Remark 3.12 and the simplifications of some proofs.

The author is supported by National Natural Science Foundation of China (No. 11901545). 


\section{References}

[Aus66] Auslander, M., Coherent functors, in Proc. Conf. Categorical Algebra, La Jolla, Calif., 1965, pp. 189-231, Springer, New York, 1966. MR0212070

[BL17] Bautista, R. and Liu, S., The bounded derived categories of an algebra with radical squared zero, J. Algebra 482 (2017), 303-345. MR3646293

[BLP13] Bautista, R., Liu, S. and Paquette, C., Representation theory of strongly locally finite quivers, Proc. Lond. Math. Soc. (3) 106 (2013), 97162. MR3020740

[BG82] Bongartz, K. and Gabriel, P., Covering spaces in representation-theory, Invent. Math. 65 (1982), 331-378. MR0643558

[Che15] Chen, X.-W., Irreducible representations of Leavitt path algebras, Forum Math. 27 (2015), 549-574. MR3334072

[EEGR09] Enochs, E., Estrada, S. and García Rozas, J. R., Injective representations of infinite quivers. Applications, Canad. J. Math. 61 (2009), 315335. MR2504018

[Gab81] Gabriel, P., The universal cover of a representation-finite algebra, in Representations of algebras, Lecture Notes in Mathematics 903, Puebla, 1980, pp. 68-105, Springer, Berlin-New York, 1981. MR0654725

[GR92] Gabriel, P. and Roiter, A. V., Representations of finite-dimensional algebras, in Algebra VIII, Encyclopaedia of Mathematical Sciences 73, pp. 1-177, Springer, Berlin, 1992. MR1239447

[Jia19] Jiao, P., Projective objects in the category of pointwise finite dimensional representations of an interval finite quiver, Forum Math. 31 (2019), 1331-1349. MR4000591

[RVdB02] Reiten, I. and Van den Bergh, M., Noetherian hereditary abelian categories satisfying Serre duality, J. Amer. Math. Soc. 15 (2002), 295366. MR1887637

[Wei94] Weibel, C. A., An introduction to homological algebra, Cambridge Studies in Advanced Mathematics 38, Cambridge University Press, Cambridge, 1994. MR1269324

Pengjie Jiao

Department of Mathematics

China Jiliang University

Hangzhou 310018

PR China

jiaopjie@cjlu.edu.cn

Received December 4, 2018

in revised form February 25, 2019 